\title{
Editorial
}

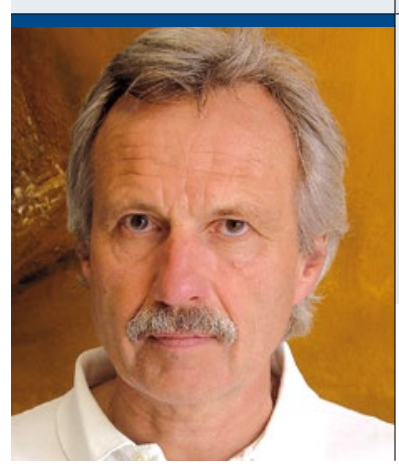

"Anschauen, abrechnen und in die Notfallambulanz der

Kliniken schicken. Ist das im Sinne eines schwer und zeitkritisch

Erkrankten?"

Dr. med. Michael Pieper (Chefredakteur)

Facharzt für Orthopädie/Unfallchirurgie, Rheumatologie

\section{Not ohne Groschen}

G esetze, die eine bessere Verzahnung von ambulant und stationär fördern sollen, scheinen das Gegenteil zu bewirken. Sie werden umgehend zum Zankapfel um die Pfründe mit dem Resultat der Divergenz, nicht der Konvergenz. Diesmal sind es die Notfallleistungen.

Die Niedergelassenen wollen die Patienten abrechnen, die mit kleinen „Wehwehchen“, die keiner dringenden Behandlung bedürfen, in die Notfallambulanzen der Kliniken strömen, anstatt den vertragsärztlichen Notdienst zu konsultieren. Dies entziehe dem vertragsärztlichen Vergütungssystem Gelder, die dann in der ambulanten Versorgung fehlen. Zumal wenn diese Patienten auch noch als Kurzzeitlieger im Krankenhaus behandelt werden. Und was geschieht mit den echten Notfällen? Anschauen, abrechnen und in die Notfallambulanz der Kliniken schicken? Ist das im Sinne eines schwer und zeitkritisch Erkrankten? Und wie trennt man die Spreu vom Weizen? Vor Ort und ad hoc? Wer besitzt diese medizinischen Fähigkeiten, ist vor Fehleinschätzungen mit haftungsrechtlichen Konsequenzen gefeit?

Im Prinzip scheint es nicht um die richtige Versorgungsstruktur zu gehen, sondern um eine Versorgungsstruktur zugunsten des vertragsärztlichen Vergütungssystems.

Zumindest herrscht monetäre Einigkeit bei KBV und GKV. Auch was die Diffamierung der Kliniken betrifft, werden alle Register gezogen: „Notaufnahmen, die als Staubsauger für unbelegte Betten fungieren“(Andreas Gassen, KBV). „Bei der Notfallversorgung geht es nicht um das Überleben von Krankenhäusern, sondern um das Überleben von Patienten" (Wulf-Dietrich Leber, GKV). Natürlich haben die Zentralinstitute und Spitzenverbände bereits die passenden Statistiken zu der Argumentation erstellt.

Nun bleibt nur noch zu klären, wer dieses „Lebenund-Tod-im-Staubsauger-Debakel“ kostenneutral löst. Portalpraxen, an denen jeder ein bisschen mitverdienen kann? Die Lösung erfolgt haftungsneutral am grünen Tisch! Von sitzfesten Zahlendrehern wird die Entlohnung von Leistungen derart konfiguriert, dass unliebsame Strukturen finanziell verhungern. Das Ganze wird dann mit dem Hinweis auf die Maxime „ambulant vor stationär" gemäß SGB V „begrünt“. Die Berliner Senatorin für Gesundheit Dilek Kolat bringt es auf den Punkt: „Die Umerziehung der Patienten hin zu bestehenden Strukturen ist aussichtslos, vielmehr müssen sich die Strukturen den geänderten Realitäten anpassen“.

Bis dato ist es nicht möglich, die Verantwortlichen von Strukturmängeln - so sie denn Schaden anrichten - zur Rechenschaft zu ziehen. Sollte je der Gesetzentwurf zur „Einführung einer strafrechtlichen Verantwortlichkeit von Unternehmen und Verbänden" aus dem NRW Justizministerium umgesetzt werden, könnte sich das ändern.

Selbst Gesundheitsminister Hermann Gröhe (CDU) scheint zerknirscht. Die Politik habe der Selbstverwaltung das Thema Vergütung der Notfallversorgung anvertraut. „Jetzt haben wir ein sehr umstrittenes Ergebnis“. Und: „Die Verabredung der Selbstverwaltung gibt Anlass, sie weiter zu beobachten“.

Überdrüssig der zerstörerischen Neiddebatte in der Ärzteschaft, könnte die Politik auf die Idee kommen, ambulant und stationär im bekannten Modell der Poliklinik zu verzahnen. Gleiche Leistung, gleiche Bezahlung! Und dann?

Ihr

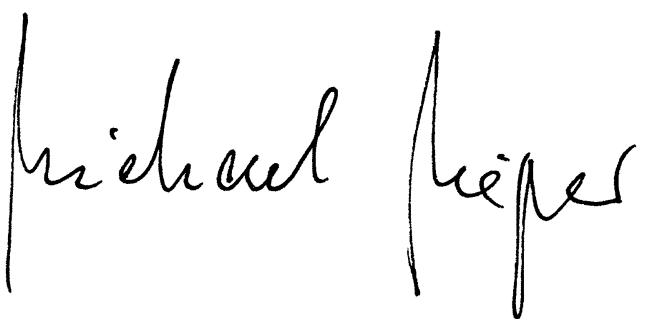

